\title{
COMPORTAMENTO DE PROGÊNIES DE CAFEEIRO ICATU ${ }^{1}$
}

\author{
Performance of coffee progenies from Icatu
}

\author{
Luiza Vasconcelos Tavares Corrêa², Antônio Nazareno Guimarães Mendes ${ }^{3}$, \\ Gabriel Ferreira Bartholo ${ }^{4}$
}

\begin{abstract}
RESUMO
Avaliar a adaptabilidade e estabilidade de progênies de cafeeiro (Coffea arabica L.) é uma etapa importante anterior à recomendação das mesmas aos cafeicultores. Com este intuito, realizou-se este trabalho, para progênies da cultivar Icatu no sul de Minas Gerais, sendo avaliada a produtividade de grãos em sacas de $60 \mathrm{~kg} / \mathrm{ha} / \mathrm{biênio.} \mathrm{Os} \mathrm{experimentos} \mathrm{foram} \mathrm{instalados} \mathrm{em} \mathrm{Machado}$ e São Sebastião do Paraíso, em estações experimentais da EPAMIG. A interação progênies x biênios x locais teve efeito significativo, justificando a utilização de metodologias de estabilidade fenotípica para classificar as progênies. Foram utilizados dados de 14 progênies de cafeeiro em 12 ambientes, considerando-se cada biênio em cada local como um ambiente. As metodologias utilizadas foram: Annicchiarico (1992) e Lin \& Binns (1988). As progênies Icatu IAC-4040-181, Icatu IAC-4040-315, Icatu IAC-4042-114, Icatu IAC-4042-222 e Icatu IAC-4045-47 se destacaram como as mais promissoras em termos de adaptabilidade e estabilidade, assim como em potencial produtivo (produtividades entre 53,68 e 59,35 sacas de $60 \mathrm{~kg} / \mathrm{ha} / \mathrm{biênio}$ ).Por outro lado, as progênies Catuaí IAC-44 e Mundo Novo IAC-379-19, dentro do grupo em estudo, foram classificadas como as mais instáveis.
\end{abstract}

Termos para indexação: Café, produtividade, adaptabilidade, estabilidade, Coffea.

\begin{abstract}
Adaptability and stability evaluations of coffee (Coffea arabica L.) progenies are an important stage prior to the recommendation of new lines. The objective this study was to evaluate bean productivity per biennium in 14 progenies from Icatu cultivar in 12 environments in southern Minas Gerais State, Brazil. The experiments were carried out in the counties of Machado and São Sebastião do Paraíso, in the experimental stations of EPAMIG. The progenies $\mathrm{x}$ biennium $\mathrm{x}$ locations interaction was significant, justifying the use of phenotypic stability methodologies for classification of progenies. Stability analyses were performed according to Annicchiarico (1992) and Lin \& Binns (1988). Progenies Icatu IAC-4040-181, Icatu IAC-4040-315, Icatu IAC-4042-114, Icatu IAC-4042-222 and Icatu IAC-4045-47 stood out as the most promising concerning adaptability and stability, as well in yield potential (productivities between 53.68 and 59.35 bags of $60 \mathrm{~kg} / \mathrm{ha} / \mathrm{biennium}$ ). On the other hand, Catuaí IAC-44 and Mundo Novo IAC-379-19, included within the progenies evaluated in this study, were classified as unstable progenies.
\end{abstract}

Index terms: Coffee, yield, adaptability, stability, Coffea.

(Recebido para publicação em 27 de abril de 2004 e aprovado em 16 de março de 2005)

\section{INTRODUÇÃO}

A cultivar Icatu é um importante marco do melhoramento do cafeeiro, pois foi um trabalho de hibridação entre duas espécies de café, Coffea arabica L. e Coffea canephora Pierre que teve como objetivo obter um material de $C$. arabica resistente à ferrugem, cujo agente causal é o fungo Hemileia vastatrix. Em Minas Gerais, desde meados da década de 1970, progênies da cultivar Icatu, introduzidas a partir de material segregante proveniente do IAC, estão sendo avaliadas e selecionadas em várias regiões produtoras do estado (MENDES \& GUIMARÃES, 1998).
A resposta diferenciada de materiais genéticos em diferentes ambientes evidencia o que se conhece como interação genótipos $\mathrm{X}$ ambientes. Uma das maneiras mais utilizadas para minimizar o efeito dessa interação seria identificar genótipos de ampla estabilidade e adaptabilidade (CRUZ \& CASTOLDI, 1991). Várias metodologias estão disponíveis para se avaliarem os materiais e classificálos quanto à estabilidade e adaptabilidade, de maneira que possam ser descartados ou recomendados para diferentes ambientes (CRUZ \& REGAZZI, 2001). Algumas dessas metodologias são: a metodologia de Lin \& Binns (1988), que avalia o desempenho de cada progênie em relação à resposta máxima em cada ambiente e a de

${ }^{1}$ Parte da dissertação do primeiro autor apresentada à Universidade Federal de Lavras (UFLA), como parte das exigências para a obtenção do título de "Mestre" em Genética e Melhoramento de Plantas.

${ }^{2}$ Agrônoma, M.Sc., Departamento de Biologia, Universidade Federal de Lavra/UFLA - Cx. P. 3037 - $37200-000$ - Lavras/MG. luizavtc@bol.com.br

${ }^{3}$ Agrônomo, Dr., Professor Departamento de Agricultura/UFLA - Cx. P. 3037, 37200-000 - Lavras/MG.

${ }^{4}$ Agrônomo, Dr., Pesquisador EPAMIG, Caixa Postal 3037 - 37200-000 - Lavras/MG 
Annicchiarico (1992), que estima o risco de adoção de determinada progênie. Mendes (1994) e Wamatu et al. (2003) são exemplos de estudos de estabilidade para a cultura do cafeeiro.

Objetivou-se com este trabalho avaliar o comportamento de algumas progênies de Icatu, consideradas como material "elite", bem como estudar a estabilidade e adaptabilidade das mesmas, no Sul de Minas Gerais.

\section{MATERIAL E MÉTODOS}

Os experimentos foram instalados em Fazendas Experimentais da EPAMIG (Empresa de Pesquisa Agropecuária de Minas Gerais), em dois locais no município de Machado (Aeroporto e Sede) e em um local no município de São Sebastião do Paraíso, ambos localizados no sul de Minas Gerais. O delineamento experimental utilizado foi o DBC (Delineamento em Blocos Casualizados), sendo os tratamentos compostos por 14 progênies de cafeeiros das cultivares Icatu, Mundo Novo e Catuaí, com quatro repetições. Cada parcela foi constituída por seis plantas. Na Tabela 1, encontra-se a relação das progênies utilizadas. Avaliouse a produção de grãos das progênies na época da colheita, no estádio de café cereja, pesando-se a produção de cada planta em separado e obtendo-se a média por parcela, em quilograma. Posteriormente, procedeu-se à conversão para a produtividade (sacas de $60 \mathrm{~kg}$ de café beneficiado/ ha).

Foram realizadas oito colheitas em cada local. Utilizou-se, para a realização das análises estatísticas e para as metodologias de estabilidade, o agrupamento das colheitas em biênios, baseando-se em trabalhos anteriores nos quais foram testados outros agrupamentos e verificou-se que o mesmo feito em biênios propiciou uma redução dos efeitos da bienalidade, aumentando a precisão (CARVALHO, 1989; MENDES, 1994). Cada biênio foi considerado uma subparcela no tempo, utilizando o esquema de parcelas subdivididas no tempo para a análise dos dados, proposto por Steel \& Torrie (1980). Após a realização da análise conjunta e a verificação da significância da interação progênies $x$ biênios $x$ locais, avaliou-se a estabilidade das progênies de acordo com as seguintes metodologias: Annicchiarico (1992) e Lin \& Binns (1988). Definiu-se como ambiente cada biênio em cada local, ou seja, 12 ambientes, como mostra a Tabela 2.

O modelo para estimação dos parâmetros de estabilidade, segundo a de Metodologia de Lin \& Binns
(1988) é dado por:

$$
P_{i}=\sum_{j=1}^{n}\left(Y_{i j}-M_{j}\right)^{2} / 2 n
$$

em que $\mathrm{P}_{\mathrm{i}}$ é o índice de estabilidade do genótipo i; $\mathrm{Y}_{\mathrm{ij}}$ é a produtividade do genótipo i no ambiente $\mathrm{j} ; \mathrm{M}_{\mathrm{j}}$ é a produtividade do genótipo com resposta máxima entre todos os genótipos no ambiente $\mathrm{j}$ e $\mathrm{n}$ é o número de ambientes. Foram estimados os valores de $\mathrm{P}_{\mathrm{i}}$ para cada progênie que se referem a uma soma de quadrados do componente de interação, tomando-se como referência o desempenho máximo de todas as progênies em cada ambiente. O modelo para estimação do parâmetro de estabilidade pela metodologia de Annicchiarico (1992) é dado por:

$$
\mathrm{I}_{\mathrm{i}}=\mathrm{Y}_{\mathrm{i} .}-\mathrm{Z}_{(1-\alpha)} \cdot \mathrm{S}_{\mathrm{i}}
$$

em que $\mathrm{I}_{\mathrm{i}}$ é o índice de confiança (\%); $\mathrm{Y}_{\mathrm{i}}$ é a média da progênie i em porcentagem; $Z$ é o percentil (1- $\alpha$ ) da função de distribuição normal acumulada e $S_{\mathrm{i}}$ é o desvio padrão dos valores percentuais. Por esta metodologia estima-se o índice de confiança $\left(I_{i}\right)$ de uma determinada progênie apresentar desempenho abaixo da média do ambiente. Será considerada ideal a progênie que apresentar o menor risco de ser adotada, isto é, deve apresentar o maior índice de confiança.

TABELA 1 - Relação das progênies avaliadas em experimentos instalados em Machado e São Sebastião do Paraíso.

\begin{aligned} & \hline $\mathbf{N}^{\circ} \multicolumn{1}{c}{$ Progênie } \\ & \hline 1 Icatu IAC -2942 \\ & 2 Icatu IAC - 2944 \\ & 3 Icatu IAC - 3282 \\ & 4 Icatu IAC $-4040-179 \\ & 5$ Icatu IAC $-4040-181 \\ & 6$ Icatu IAC $-4040-315 \\ & 7$ Icatu IAC $-4042-114 \\ & 8$ Icatu IAC $-4042-222 \\ & 9$ Icatu IAC $-4045-47 \\ & 10$ Icatu IAC $-4228-101 \\ & 11$ Icatu IAC -4782 \\ & 12 Catuaí IAC -44 \\ & 13 Mundo Novo IAC $-379-19 \\ & 14$ Mundo Novo IAC $-388-17 \\ &$\hline\end{aligned}


TABELA 2 - Relação dos ambientes utilizados para a avaliação da estabilidade de acordo com a combinação de locais e biênios.

\begin{tabular}{lllll}
\hline \multirow{2}{*}{\multicolumn{1}{c}{ Locais }} & \multicolumn{4}{c}{ Biênios } \\
\cline { 2 - 5 } & I & II & III & IV \\
\hline Machado/Aeroporto & 1 & 2 & 3 & 4 \\
Machado/Sede & 5 & 6 & 7 & 8 \\
S. S. Paraíso & 9 & 10 & 11 & 12 \\
\hline
\end{tabular}

\section{RESULTADOS E DISCUSSÃO}

De acordo com a análise conjunta (Tabela 3), as fontes de variação biênios e as interações progênies $x$ biênios e progênies $\mathrm{x}$ biênios $\mathrm{x}$ locais tiveram efeito significativo, justificando, portanto a utilização de metodologias que classifiquem as progênies de acordo com a adaptabilidade e estabilidade das mesmas. Os coeficientes de variação (CV) obtidos estão coerentes com outros trabalhos em que se utilizou o mesmo tipo de dado (produção em biênios), indicando boa precisão experimental (CARVALHO, 1989; MENDES, 1994).

TABELA 3 - Resumo da análise de variância conjunta no esquema de parcelas subdivididas no tempo para produtividade de café beneficiado, em sacas de $60 \mathrm{~kg} / \mathrm{ha} /$ biênio, nos locais Machado/Aeroporto; Machado/Sede e São Sebastião do Paraíso.

\begin{tabular}{lcrll}
\hline \multicolumn{1}{c}{ FV } & GL & QM & \\
\hline Blocos (B) / (Locais) & 9 & 830,62 & \\
Progênies (P) & 13 & 1049,50 & NS \\
Locais (L) & 2 & 110823,98 & NS \\
P x L & 26 & 466,03 & NS \\
Erro (a) & 117 & 294,42 & \\
\hline Biênios (C) & 3 & 103400,01 & $* *$ \\
B x C / (L) & 27 & 264,51 & $* *$ \\
L x C & 6 & 46704,22 & $* *$ \\
P x C & 39 & 569,42 & $* *$ \\
P x C x L & 78 & 201,61 & $* *$ \\
Erro (b) & 351 & 111,79 & \\
\hline CV a (\%) & 33,16 & & \\
CV b (\%) & 20,43 & & \\
\hline
\end{tabular}

(NS) e (**) Não-significativo e significativo ao nível de $1 \%$ de probabilidade pelo teste de $\mathrm{F}$.
Na Tabela 4, observa-se a produtividade média de cada progênie por biênio, nos três locais, sendo as progênies Icatu IAC-4040-181, Icatu IAC-4040-315, Icatu IAC-4042-114, Icatu IAC-4042-222, Icatu IAC-4045-47 e Icatu IAC-4228-101 as mais produtivas.

TABELA 4 - Produtividade média por biênio de café beneficiado, em sacas de $60 \mathrm{~kg} / \mathrm{ha}$, nos locais Machado/ Aeroporto; Machado/Sede e São Sebastião do Paraíso.

\begin{tabular}{lc}
\hline \multicolumn{1}{c}{ Progênies } & Média/Biênio \\
\hline Icatu IAC - 2942 & $50,32 \mathrm{~b}$ \\
Icatu IAC - 2944 & $51,16 \mathrm{~b}$ \\
Icatu IAC - 3282 & $47,17 \mathrm{~b}$ \\
Icatu IAC - 4040 - 179 & $50,21 \mathrm{~b}$ \\
Icatu IAC - 4040 - 181 & $57,29 \mathrm{a}$ \\
Icatu IAC - 4040 - 315 & $59,35 \mathrm{a}$ \\
Icatu IAC - 4042 - 114 & $57,47 \mathrm{a}$ \\
Icatu IAC - 4042 - 222 & $53,68 \mathrm{a}$ \\
Icatu IAC - 4045 - 47 & $55,96 \mathrm{a}$ \\
Icatu IAC - 4228 - 101 & $52,93 \mathrm{a}$ \\
Icatu IAC - 4782 & $51,46 \mathrm{~b}$ \\
Catuaí IAC - 44 & $48,17 \mathrm{~b}$ \\
Mundo Novo IAC - 379 - 19 & $43,89 \mathrm{~b}$ \\
Mundo Novo IAC - 388 -17 & $45,45 \mathrm{~b}$ \\
\hline
\end{tabular}

Médias seguidas pela mesma letra não diferem entre si ao nível de 5\% de probabilidade, pelo teste de Scott e Knott.

Na Tabela 5, estão dispostos os parâmetros de acordo com a metodologia de Lin \& Binns (1988), que avalia o desempenho de cada progênie em relação à produtividade máxima em cada ambiente. Uma grande vantagem deste método é que a medida geral de superioridade consiste de um único parâmetro, simplificando sobremaneira o processo de classificação. Os outros parâmetros estimados por este método são decomposições das estimativas de $\mathrm{P}_{\mathrm{i}}$ e são utilizados para indicar a adaptabilidade geral dos genótipos (LIN \& BINNS, 1988). Neste sentido, a progênie Icatu IAC4040-315 foi a mais estável, apresentando a menor estimativa do parâmetro que avalia estabilidade $\left(\mathrm{P}_{\mathrm{i}}\right)$ e foi a única que não diferiu significativamente, ao nível de 5\% de probabilidade, do desempenho máximo obtido, contribuindo com apenas $2,78 \%$ da interação. As progênies Icatu IAC-4040-181, Icatu IAC-4042-114 e Icatu IAC-4045-47 apresentaram valores desejáveis de $\mathrm{P}_{\mathrm{i}} \mathrm{e}$ também foram as que menos contribuíram para a interação,

Ciênc. agrotec., Lavras, v. 30, n. 4, p. 618-622, jul./ago., 2006 
sendo considerados materiais promissores. A progênie Catuaí IAC-44, por outro lado, foi a que obteve maior valor de $\mathrm{P}_{\mathrm{i}}$ e a que apresentou maior contribuição para a interação, considerada, portanto, o pior material, quanto à estabilidade de produção.

Pela metodologia de Annicchiarico (1992), estima-se o índice de confiança, ou seja, o risco de sucesso na escolha de uma determinada progênie, associado a um nível de significância $(\alpha)$, neste caso de 0,25 . De acordo com a Tabela 6, observa-se que as progênies Icatu IAC-4040-181, Icatu IAC-4040-315, Icatu IAC-4042-114, Icatu IAC-4042-222 e Icatu IAC-4045-47 foram as que mais se destacaram, obtendo os maiores valores de $\mathrm{I}_{\mathrm{i}}$, sendo que as progênies Icatu IAC4040-181, Icatu IAC-4040-315, Icatu IAC-4042-222 e Icatu IAC-4045-47 apresentaram, com 75\% de confiança, os menores riscos de possuírem comportamento abaixo da média do ambiente, de 8,13\%,0,89\%, 0,74\% e 5,66\%, respectivamente. A progênie Icatu IAC-4042-114 apresentou com $75 \%$ de confiança, a probabilidade de ter desempenho de 7,63\% superior à média ambiental, sendo, portanto, a de melhor comportamento por essa metodologia. A progênie Mundo Novo IAC-379-19, por sua vez, foi a que apresentou menor valor de $I_{i}$, apresentando o risco de $27,37 \%$ de se comportar abaixo da média dos ambientes.

TABELA 5 - Estimativa dos parâmetros de estabilidade, segundo a metodologia proposta por Lin \& Binns (1988), para produtividade de café beneficiado, em sacas de $60 \mathrm{~kg} / \mathrm{ha} / \mathrm{biênio.}$

\begin{tabular}{lcccc}
\hline \multicolumn{1}{c}{ Progênie } & $\mathbf{P}_{\mathbf{i}}^{*}$ & $\begin{array}{c}\text { Efeito } \\
\text { Genético }\end{array}$ & $\begin{array}{c}\text { Desvio da } \\
\text { Interação }\end{array}$ & $\begin{array}{c}\text { Interação } \\
(\%)\end{array}$ \\
\hline Icatu IAC - 2942 & 1190,87 & 590,19 & 600,68 & 17,39 \\
Icatu IAC - 2944 & 842,66 & 533,17 & 309,49 & 8,96 \\
Icatu IAC - 3282 & 1002,70 & 825,94 & 176,76 & 5,12 \\
Icatu IAC - 4040 - 179 & 751,06 & 597,83 & 153,23 & 4,44 \\
Icatu IAC - 4040 - 181 & 322,67 & 208,08 & 114,59 & 3,32 \\
Icatu IAC - 4040 - 315 & 228,74 & 132,57 & 96,17 & 2,78 \\
Icatu IAC - 4042 - 114 & 286,77 & 200,60 & 86,17 & 2,49 \\
Icatu IAC - 4042 - 222 & 563,73 & 381,47 & 182,25 & 5,28 \\
Icatu IAC - 4045 - 47 & 366,89 & 265,96 & 100,93 & 2,92 \\
Icatu IAC - 4228 - 101 & 714,75 & 423,70 & 291,05 & 8,43 \\
Icatu IAC - 4782 & 696,33 & 514,46 & 181,86 & 5,26 \\
Catuaí IAC - 44 & 1366,85 & 747,23 & 619,62 & 17,93 \\
Mundo Novo IAC - 379 -19 & 1350,68 & 1114,08 & 236,61 & 6,85 \\
Mundo Novo IAC - 388 -17 & 1277,39 & 972,26 & 305,13 & 8,83 \\
\hline
\end{tabular}

* Ponto de corte $($ cutoff point $)=284,84$. $\mathrm{P}_{\mathrm{i}}$ menor que este valor não difere significativamente do máximo $(\mathrm{p}<0,05)$. 
TABELA 6 - Estimativa do índice de confiança, segundo o método proposto por Annicchiarico (1992), para produtividade de café beneficiado, em sacas de $60 \mathrm{~kg} / \mathrm{ha} / \mathrm{biênio.}$

\begin{tabular}{|c|c|c|c|}
\hline Progênie & $\mathbf{Y}_{\mathbf{i}}$ & $\mathbf{S}_{\mathbf{i}}$ & $\mathbf{I}_{\mathrm{i}}{ }^{*}$ \\
\hline Icatu IAC - 2942 & 105,55 & 28,04 & 86,64 \\
\hline Icatu IAC - 2944 & 94,30 & 23,85 & 78,21 \\
\hline Icatu IAC - 3282 & 89,28 & 11,11 & 81,79 \\
\hline Icatu IAC - 4040 - 179 & 92,58 & 20,24 & 78,93 \\
\hline Icatu IAC - 4040 - 181 & 108,91 & 25,26 & 91,87 \\
\hline Icatu IAC - 4040 - 315 & 111,35 & 18,15 & 99,11 \\
\hline Icatu IAC - $4042-114$ & 113,46 & 8,65 & 107,63 \\
\hline Icatu IAC - 4042 - 222 & 107,11 & 11,64 & 99,26 \\
\hline Icatu IAC - $4045-47$ & 109,83 & 22,96 & 94,34 \\
\hline Icatu IAC - 4228 - 101 & 102,62 & 19,58 & 89,41 \\
\hline Icatu IAC - 4782 & 91,69 & 22,40 & 76,58 \\
\hline Catuaí IAC - 44 & 101,80 & 33,32 & 79,32 \\
\hline Mundo Novo IAC - 379 - 19 & 82,48 & 14,60 & 72,63 \\
\hline Mundo Novo IAC - 388 -17 & 89,03 & 13,72 & 79,78 \\
\hline
\end{tabular}

Nível de significância adotado $\alpha=0,25$.

\section{CONCLUSÕES}

As progênies Icatu IAC-4040-181, Icatu IAC4040-315, Icatu IAC-4042-114, Icatu IAC-4042-222 e Icatu IAC-4045-47 mostram-se como as mais promissoras, tanto em termos de adaptabilidade e estabilidade quanto para potencial produtivo. As progênies Catuaí IAC-44 e Mundo Novo IAC-379-19 classificam-se sempre, no grupo de progênies estudado, como os piores genótipos quanto à estabilidade e associados a uma baixa produtividade.

As melhores progênies apontadas pelos parâmetros estimados em cada metodologia utilizada foram a Icatu IAC4040-315, de acordo com Lin \& Binns, (1988) e a Icatu IAC4042-114, de acordo com Annicchiarico (1992).

\section{REFERÊNCIAS BIBLIOGRÁFICAS}

ANNICCHIARICO, P. Cultivar adaptation and recommendation from alfafa trials in Northern Italy. Journal of Genetics and Breeding, Rome, v. 46, n. 3, p. 269-278, Sept. 1992.

CARVALHO, S. P. Metodologias de avaliação do desempenho de progênies do cafeeiro (Coffea arabica L.). 1989. 68 f. Dissertação (Mestrado em Genética e Melhoramento de Plantas) - Universidade Federal de Lavras, Lavras, 1989.
CRUZ, C. D.; CASTOLDI, F. L. Decomposição da interação genótipos x ambientes em partes simples e complexa. Revista Ceres, Viçosa, v. 38, n. 219, p. 422-430, 1991.

CRUZ, C. D.; REGAZZI, A. J. Modelos biométricos aplicados ao melhoramento genético. 2. ed. Viçosa: UFV, 2001. 390 p.

LIN, C. S.; BINNS, M. R. A superiority measure of cultivar performance for cultivar $\mathrm{x}$ location data. Canadian Journal of Plant Science, Ottawa, v. 68, n. 1, p. 193-198, Jan./Mar. 1988.

MENDES, A. N. G. Avaliação de metodologias empregadas na seleção de progênies do cafeeiro (Coffea arabica L.) no estado de Minas Gerais. 1994. 167 f. Tese (Doutorado em Fitotecnia) - Universidade Federal de Lavras, Lavras, 1994.

MENDES, A. N. G.; GUIMARÃES, R. J. Genética e melhoramento do cafeeiro. Lavras: UFLA/FAEPE, 1998. 99 p.

STEEL, R. G.; TORRIE, J. K. Principles and procedures of statistics: a biometrical approach. 2. ed. Tokyo: McGraw-Hill, 1980. 633 p.

WAMATU, J. N.; THOMAS, E.; PIEPHO, H. P. Responses of different arabica coffee (Coffea arabica L.) clones to varied environment conditions. Euphytica, Wageningen, v. 129, n. 2, p. 175-182, 2003. 